\title{
TEORÍA DE BILL MUNDY EN EL MECANIZADO DE COBRE CON BROCAS Y MACHOS ESPECIALES
}

\section{BILL MUNDY THEORY. SPECIAL DRILLS AND TAPS TOOLS IN COPPER MACHINING}

\author{
Alejandro Colque C. ${ }^{1} \quad$ Alfredo Coria C. ${ }^{1} \quad$ Juan M. Godoy R. $^{1} \quad$ Jorge Vergara $^{1}{ }^{1}$ \\ Recibido el 17 de abril de 2007, aceptado el 15 de enero de 2008 \\ Received: April 17, 2007 Accepted: January 15, 2008
}

\begin{abstract}
RESUMEN
El mecanizado del cobre, debido a su fluidez, produce una viruta plástica y continua que afecta la calidad superficial de las piezas mecanizadas. El método desarrollado por el ingeniero norteamericano Bill Mundy permite obtener el mejor ángulo de corte para mecanizar un material. El taladrado y el roscado con machos son dos procesos de mecanizado que tienen aspectos en común. Diseños de nuevas herramientas, nuevos materiales y técnicas de mecanizado, mejorías en la lubricación, diferentes velocidades de corte y avances son variables que se deben estudiar para obtener una menor potencia, mayor duración de la herramienta y buen acabado superficial.

En este trabajo probetas de cobre fueron ensayadas a tracción y se determinó experimentalmente el ángulo efectivo de corte, de acuerdo con la teoría de Bill Mundy. Posteriormente se fabricaron nuevas probetas que fueron perforadas con brocas tipo $\mathrm{H}, \mathrm{N}$ y W, con refrigeración externa, refrigeración interna y en seco. Se usaron machos de máquina de dos y tres hélices. En cada perforación se midió la velocidad de rotación, avance, fuerza de corte, momento torsor y potencia necesaria.

Se verificó que en el taladrado de cobre un ángulo mayor de la hélice favorece el desmenuzado de la viruta y disminuye el momento torsor. Brocas con refrigeración interna pueden ser utilizadas con mayores velocidades y muestran una mejor calidad superficial del perforado. Las brocas y machos con un ángulo de ataque próximo al ángulo calculado experimentalmente por la teoría de Bill Mundy muestran un buen comportamiento en el mecanizado y menor consumo de potencia.
\end{abstract}

Palabras clave: Cobre, mecanizado, brocas, machos especiales.

\begin{abstract}
Copper drilling produces plastic and continuous chip. Bill Mundy a North American engineer developed a method who allows determines experimentally the best cutting angle material machining. Drilling and threading processes has several aspects in common. New tools design, new materials, new techniques, new lubrication and cooling improvement, involve new different cutting rate and feed, parameters that should be necessarily studied to power, and time operation reduction for increase the edge cutting life and improving the finish surface.

In this work tensile strength test was made in copper, through the results the cutting angle was found according the Bill Mundy theory. Lately new test bars were cut and were perforated with drills type $H, N$ and $W$, with external refrigeration, internal refrigeration, and dry. Tap for machining tools with two or three helixes were used. For each test was registered the cutting speed, feed, cutting forces, torsor moment and power consumption.

It was verified that in the copper drilling, that a bigger helix angle facilitates the shredded of the chip and reduces the torsor moment. Drills with internal cooling system can work with higher cutting speeds improving surface quality than tools with externally cooling system. Drills and taps with angle near the experimentally calculated according to the Bill Mundy theory show a good behaviour in the drilled process and also small power consumption.
\end{abstract}

Keywords: Copper, drilling, tools, taps and tap form.

1 Escuela Universitaria de Ingeniería Mecánica. Universidad de Tarapacá. 18 Septiembre 2222. Arica, Chile. E-mail: jvergara@uta.cl, jmgodoy@uta.cl 


\section{INTRODUCCIÓN}

Los materiales usados en la fabricación de herramientas de corte muestran cada día un gran mejoramiento. Bill Mundy recomienda "deberíamos mirar el metal para observar cómo él desea ser cortado" [1]. La geometría inadecuada de la herramienta de corte provoca daños al metal de origen, creando una superficie difícil de trabajar y con elevados esfuerzos residuales. Su teoría está relacionada con el ángulo de ataque. "El ángulo de ataque efectivo calculado por este método, permite a la herramienta de corte generar una falla de viruta a $45^{\circ}$ del metal de origen" [1]. La geometría del filo de corte determinada por este procedimiento es aplicada a todos los rangos de herramientas para torneado, fresado, taladrado, roscado, escariado y trepanado. El ángulo de ataque efectivo para una herramienta de corte de metal se determina experimentalmente en la ecuación (1) [1]:

$$
\cos \cdot \gamma=\left(\frac{l_{i}}{l_{f}}\right) \cdot \cos \cdot \beta
$$

$\gamma=$ ángulo de ataque efectivo,

$l i=$ largo inicial de la probeta de tracción antes de la ruptura,

lf = largo después de la ruptura,

$\beta=$ ángulo de cuello en el área de falla.

El ángulo de ataque efectivo siempre da un resultado positivo para la mayoría de los metales. Este ángulo se encuentra entre $25^{\circ}$ y $55^{\circ}$. Alrededor de $3 / 4$ de los metales comúnmente mecanizados se agrupan en los $35^{\circ}$. En la práctica existe una gran área que es frecuentemente desechada por la teoría general "porque la porción de la curva coseno en la cual se trabaja absorbe una gran parte de las diferencias dimensionales" [1].

\section{DESARROLLO}

\section{Equipos}

La composición química de los cobres y latones fue determinada en un espectrofotómetro de emisión de la Empresa Fundición Neptuno-Iquique.

El ensayo de tracción de las probetas de cobre fue realizado en una Máquina Universal de Ensayos Kratos de 20 toneladas.

La medición del ángulo del cuello de ruptura de las probetas y los ángulos de corte de las herramientas se realizó en el Gran Microscopio de Herramientas Carl Zeiss.
El tamaño de grano fue determinado en un microscopio Nikon, modelo Optiphot adaptado con una cámara de video, un PC y una tarjeta de video, para adquisición de imágenes.

La verificación de la circularidad de los agujeros fue realizada en una máquina de 3 coordenadas marca Mitutoyo, Modelo BRT504.

La rugosidad superficial de las piezas se midió con un rugosímetro Hommel Tester.

Los parámetros del proceso se determinaron en un Centro de Mecanizado HAAS VF-OE, con el apoyo de un dinamómetro piezoeléctrico Kistler y un sistema de adquisición de datos DAS 802, instalado en un PC empleando los programas TESTPOINT y Exact [2].

Para el cálculo de la potencia, el dinamómetro piezoeléctrico fue calibrado con una llave de torque de 7,4 N.m. La potencia se determina mediante la ecuación (2).

$$
P S=\frac{M \cdot S \cdot 2 \cdot \pi}{60}[W]
$$

$\mathrm{M}=$ momento $[\mathrm{N} \cdot \mathrm{m}]$.

$\mathrm{S}=$ velocidad giro herramienta $[\mathrm{rpm}]$.

En esta ecuación no se ha considerado el momento en el eje vertical y el rendimiento de la máquina. Se utilizará como Potencia total el valor calculado Ps multiplicado por 1,5 como lo recomienda la literatura, ecuación (3) [3].

$$
\text { Ptotal }=1,5 \operatorname{Ps}[W]
$$

El afilado de las brocas fue realizado en la Afiladora óptica marca Optima.

Las brocas utilizadas para confeccionar las roscas M-10, fueron de 8,5 mm de diámetro:

\section{Brocas}

Las brocas utilizadas en este trabajo fueron seleccionadas del catálogo de la Titex Plus [4].

Broca helicoidal Titex Plus tipo H modelo A 1212. La broca helicoidal tipo $\mathrm{H}$ fue fabricada de acero rápido sinterizado sin recubrimiento y posee mango cilíndrico. Esta herramienta se puede aplicar en materiales quebradizos de viruta corta como es el latón MS 62 (UNS-C86200), aleaciones de magnesio, Zamak, plásticos (plexiglás en agujeros de poca profundidad), por lo tanto, este tipo de broca no es recomendada para el taladrado en cobre. 
Broca helicoidal Titex Plus tipo N modelo A 1211. La broca helicoidal tipo $\mathrm{N}$ fue fabricada de acero rápido sinterizado y posee mango cilíndrico. Esta broca es recomendada para aceros, fundición gris, acero sinterizado, plata alemana y aleaciones aluminio-silicio.

Broca helicoidal Titex Plus tipo W modelo A 1213. La broca helicoidal tipo $\mathrm{W}$ fue fabricada de acero rápido sin recubrimiento y posee mango cilíndrico. Es recomendada para materiales blandos como aluminio, cobre, cinc, plásticos blandos.

Broca Mega Jet Titex Plus modelo A6292 TIN. Esta broca de alto rendimiento con refrigeración interna posee una geometría especial para un control óptimo de la viruta y su evacuación en materiales de viruta larga. Tiene un recubrimiento de Nitruro de Titanio. Puede ser asociada al tipo N.

Broca Garant 12235. Esta es una broca de metal duro de alto rendimiento con conductos de refrigeración interna de mango cilíndrico y diámetro $9,3 \mathrm{~mm}$, con recubrimiento de Nitruro de Titanio. Su uso está relacionado con el proceso de roscado por laminación. Esta broca posee características geométricas distintas a las convencionales.

La figura 1 muestra las brocas Titex plus A 1211 (tipo N), A 1212 (tipo H), A 1213 (tipo W), A 6292 tipo Mega Jet (asociada tipo N) y Garant 12235 (para roscado por laminación), ordenadas de izquierda a derecha respectivamente.

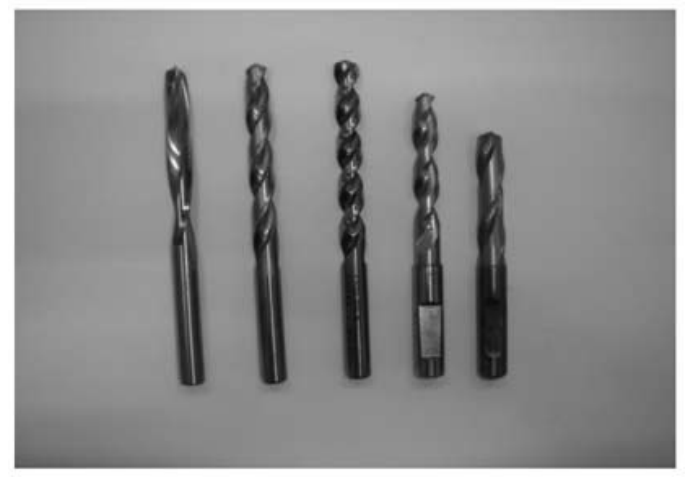

Figura 1. Brocas Titex plus y Garrant.

\section{Machos}

Los machos utilizados, que se muestran en la figura 2, fueron usados para realizar una rosca M-10 en cobre, con desprendimiento de viruta, en un agujero ciego con refrigeración externa y con una tolerancia ISO H6/h6. En estos ensayos se utilizaron machos Titex Plus M-10, con remoción de viruta, tipos B 1233, B 1234, y B 1251 de tres hélices, y el macho B 1235 con dos hélices. Además se utilizó un macho de roscado por laminación M-10, marca Emuge.

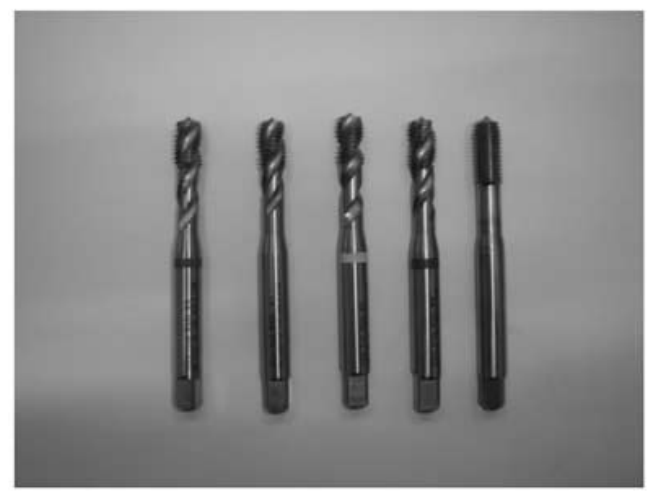

Figura 2. Machos seleccionados mediante catálogo.

\section{Materiales}

Se utilizaron barras de cobre ETP, de $50 \mathrm{~mm}$ de diámetro. Se fabricaron probetas proporcionales de $6 \mathrm{~mm}$ de diámetro, para el ensayo de tracción y una serie de probetas de 50 $\mathrm{mm}$ de diámetro por $40 \mathrm{~mm}$ de altura para las experiencias de taladrado y roscado.

\section{RESULTADOS}

\section{Ensayo de tracción en las probetas de cobre}

Se fabricaron probetas proporcionales con $6 \mathrm{~mm}$ de diámetro y fueron ensayadas en la Máquina Universal de Ensayos modelo Kratos. En la tabla 1 se indican las variables medidas en el ensayo de tracción. Se obtuvo un ángulo medio de ataque efectivo $\gamma$ de $43^{\circ}$.

Tabla 1. Ensayo de tracción y ángulo de ataque.

\begin{tabular}{|c|c|c|}
\hline Variables & Probeta 1 & Probeta 2 \\
\hline $\begin{array}{c}\text { Diámetro Inicial } \\
\text { [mm] }\end{array}$ & 6 & 6 \\
\hline $\begin{array}{c}\text { Longitud Inicial } \\
{[\mathbf{m m}]}\end{array}$ & 30 & 30 \\
\hline $\begin{array}{c}\text { Longitud Final } \\
{[\mathbf{m m}]}\end{array}$ & 39 & 39 \\
\hline $\begin{array}{c}\text { Resistencia Tracción } \\
{[\text { MPa] }}\end{array}$ & 250 & 250 \\
\hline Ángulo $\beta$ & $17,5^{\circ}$ & $19^{\circ}$ \\
\hline Ángulo de Ataque $\gamma$ & $42,8^{\circ}$ & $43,3^{\mathbf{o}}$ \\
\hline
\end{tabular}




\section{Taladrado del cobre}

Medición de los ángulos de corte de las brocas. Se midieron con el Gran Microscopio de Herramientas Carl Zeiss, los ángulos aparentes de las brocas utilizadas. Estas mediciones fueron realizadas a una temperatura de $20{ }^{\circ} \mathrm{C}$. Los resultados de las mediciones se muestran en la tabla 2 .

Tabla 2. Ángulos de corte de las brocas.

\begin{tabular}{|c|c|c|c|c|}
\hline \multirow{2}{*}{ BROCA } & \multicolumn{4}{|c|}{ ÁNGULOS } \\
\cline { 2 - 5 } & $\begin{array}{c}\text { Incidencia } \\
\alpha\end{array}$ & $\begin{array}{c}\text { Filo } \\
\beta\end{array}$ & $\begin{array}{c}\text { Ataque } \\
\gamma\end{array}$ & $\begin{array}{c}\text { Punta } \\
\text { E }\end{array}$ \\
\hline $\begin{array}{c}\mathbf{A} \\
\mathbf{1 2 1 2}(\mathbf{H})\end{array}$ & $\mathbf{4}^{\mathbf{o}} \mathbf{2 2}$, & $\mathbf{7 1}^{\mathbf{o}} \mathbf{2 5}$, & $\mathbf{1 4}^{\mathbf{0}} 13$ & $\mathbf{1 1 8}^{\circ}$ \\
\hline $\begin{array}{c}\mathbf{A} \\
\mathbf{1 2 1 1}(\mathbf{N})\end{array}$ & $6^{\circ} 20^{\prime}$ & $54^{\circ} 20^{\prime}$ & $29^{\circ} 20^{\prime}$ & $118^{\circ}$ \\
\hline $\begin{array}{c}\mathbf{A} \\
\mathbf{1 2 1 3}(\mathbf{W})\end{array}$ & $3^{\circ} 35^{\prime}$ & $43^{\circ} 05^{\prime}$ & $43^{\circ} 20^{\prime}$ & $130^{\circ}$ \\
\hline $\begin{array}{c}\text { MegaJet } \\
\text { A6292 }\end{array}$ & $8^{\circ} 55^{\prime}$ & $45^{\circ} 00^{\prime}$ & $36^{\circ} 05^{\prime}$ & $130^{\circ}$ \\
\hline $\begin{array}{c}\text { Garant } \\
\mathbf{1 2 2 3 5}\end{array}$ & $10^{\circ} 12^{\prime}$ & $52^{\circ} 23^{\prime}$ & $27^{\circ} 25^{\prime}$ & $140^{\circ}$ \\
\hline
\end{tabular}

La broca A 1213 (tipo W), con un ángulo de ataque efectivo de $\gamma=43^{\circ}$, se ajusta perfectamente con el ángulo efectivo de ataque obtenido a partir de las probetas de cobre de acuerdo con la teoría de Bill Mundy. La broca Mega Jet A 6292 tiene un ángulo $\gamma=36^{\circ}$. La broca A 1211 (tipo $\mathrm{N})$ tiene un ángulo $\gamma=29^{\circ}$, mientras que la broca $\mathrm{A}$ 1212 (tipo H) tiene un ángulo $\gamma=14^{\circ}$. Cabe señalar que la broca Garant 12235 con un ángulo de $\gamma=27^{\circ}$ posee filos especiales, los que influyen directamente en la eficiencia del proceso, lo que requiere de un análisis especial.

Taladrado sin refrigeración. Las brocas Titex Plus con diámetro 8,5 mm, Modelos A 1212 tipo H, A 1211 tipo N y A 1213 tipo W fueron utilizadas en el taladrado de las probetas de cobre, sin refrigeración, con tres avances diferentes y diferentes velocidades de corte. La figura 3 muestra las curvas de potencia versus avance, para una velocidad de giro de 1.123 [rpm], que corresponde a una velocidad de corte de 30 [m/min].

La broca tipo H que necesitó mayor potencia se quebró con el avance de 0,20 [mm/rev]. La broca tipo W muestra el menor consumo de potencia, lo que se manifiesta nuevamente a mayores velocidades de corte, como $\mathrm{Vc}=$ $40[\mathrm{~m} / \mathrm{min}]$ y $\mathrm{Vc}=50[\mathrm{~m} / \mathrm{min}]$.

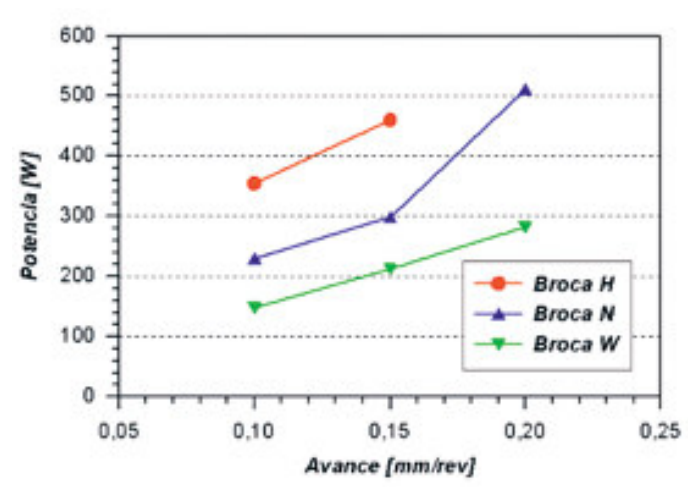

Figura 3. Potencia v/s Avance sin refrigeración, para $\mathrm{Vc}=30[\mathrm{~m} / \mathrm{min}]$.

Taladrado con refrigeración externa. Las brocas Titex Plus anteriormente indicadas fueron utilizadas en el taladrado de las probetas de cobre con refrigeración externa. Se utilizaron diversas velocidades de corte, con tres avances diferentes. La figura 4 muestra las curvas de potencia versus avance, para una velocidad de giro de 1.498 [rpm], que corresponde a una velocidad de corte de $40[\mathrm{~m} / \mathrm{min}]$.

Los gráficos correspondientes, para velocidades de corte superiores a la indicada, mostraron las mismas tendencias donde el menor consumo de potencia correspondió a la broca tipo W.

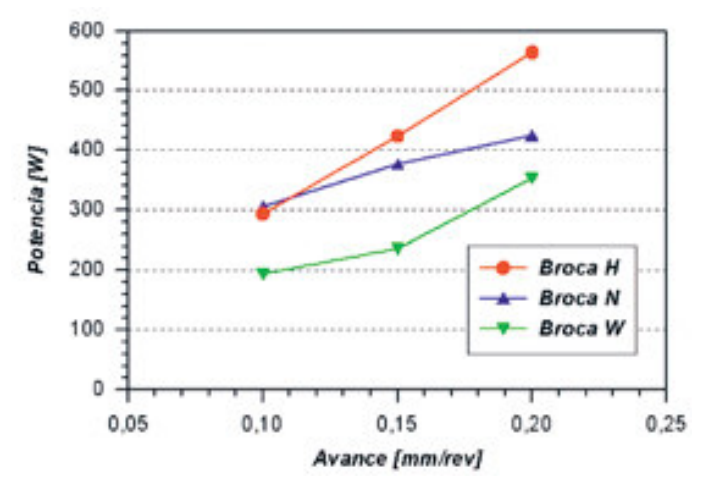

Figura 4. Potencia v/s Avance con refrigeración interna, para $\mathrm{Vc}=40 \mathrm{~m} / \mathrm{min}$.

Taladrado con refrigeración interna. Los ensayos con refrigeración interna fueron realizados con la broca Titex Plus Modelo A 6292 TiN, tipo Mega Jet. La figura 5 muestra los valores de potencia versus avance para distintas velocidades de corte. Cuando aumentan las rotaciones o velocidad de corte, la potencia aumenta proporcionalmente. 

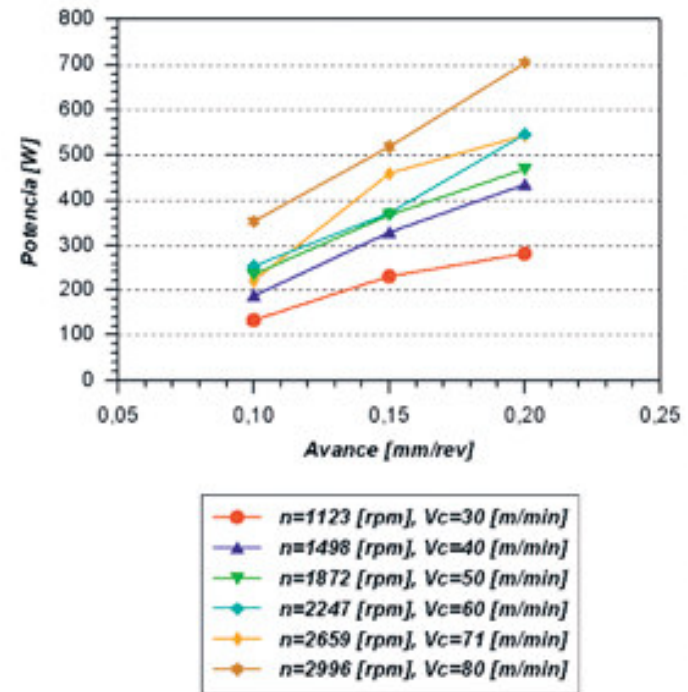

Figura 5. Potencia v/s Avance con refrigeración interna.

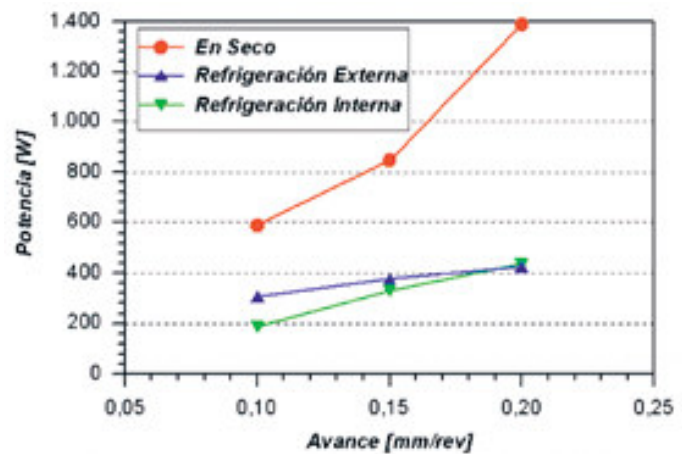

Figura 6. Potencia v/s Avance para diferentes tipos de refrigeración, broca tipo $\mathrm{N}$.

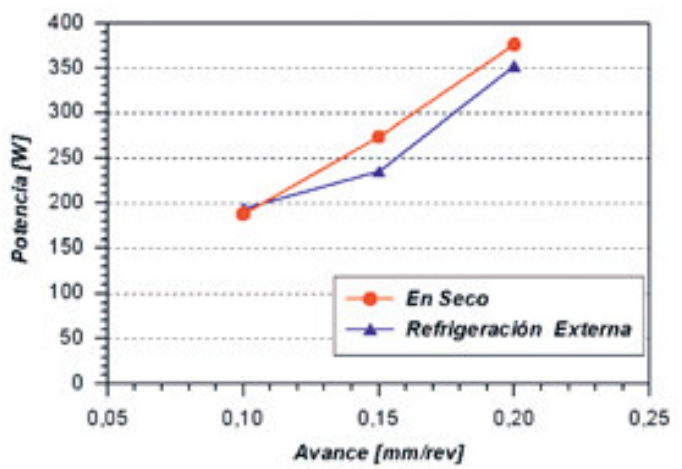

Figura 7. Potencia v/s Avance, para diferentes tipos de refrigeración, broca tipo $\mathrm{W}$.

Broca tipo $N$. Taladrado sin refrigeración y con refrigeración externa e interna. En los ensayos sin y con refrigeración externa se utilizaron las brocas Titex Plus Mod A 1211. Para la refrigeración interna se utilizó la broca Modelo A 6292 tipo Mega Jet.
La figura 6 muestra el consumo de potencia versus avance, para 1.498 [rpm], que equivale a una $\mathrm{Vc}=40[\mathrm{~m} / \mathrm{min}]$.

Al aumentar el avance, el consumo de potencia es similar para las brocas con refrigeración interna y externa. Para bajas velocidades de corte, el consumo de potencia es menor para la broca con refrigeración interna. Para una velocidad de corte de 50 [m/min], el consumo de potencia para las brocas con refrigeración externa e interna sigue siendo similar. La broca sin refrigeración muestra un alto consumo de potencia al aumentar el avance.

Broca tipo W. Taladrado sin y con refrigeración externa. Estos ensayos fueron realizados sin y con refrigeración externa, se utilizaron las brocas Titex Plus Modelo A 1213. La figura 7 muestra el consumo de potencia versus avance para $1.498[\mathrm{rpm}]$, lo que equivale a una $\mathrm{Vc}=40$ [m/min].

Se observa que la potencia es similar para un avance de 0,10 [mm/rev]. Para los avances de 0,15 y 0,20 [mm/rev] el consumo de potencia es menor para la broca refrigerada externamente. Para velocidades de corte de 30 [m/min], y $50[\mathrm{~m} / \mathrm{min}]$ el consumo de potencia es similar para el taladrado con y sin refrigeración.

Virutas arrancadas en el proceso. Las brocas tipo $\mathrm{H}$, generan una viruta larga en espiral que se enrosca en la broca. $\mathrm{La}$ broca tipo $\mathrm{N}$ y $\mathrm{W}$ generan una viruta desmenuzada que no ocasiona problemas a la broca. La broca Modelo A 6292 tipo Mega Jet genera una viruta en espiral larga, de bordes lisos que tiende a enroscarse, pero a velocidades altas sale con gran facilidad en forma radial y violentamente.

Rugosidad de los Agujeros. En la tabla 3 se observan los mejores valores de la rugosidad media para los agujeros realizados con las diferentes brocas, en función a los mejores datos de corte. La broca tipo W entregó la rugosidad media de menor calidad superficial, mientras que las brocas tipo $\mathrm{H}, \mathrm{N}$ y con refrigeración interna muestran una calidad superficial superior y una menor rugosidad.

Tabla 3. Rugosidad Media Ra de las brocas.

\begin{tabular}{|c|c|c|c|c|}
\hline Brocas & $\begin{array}{c}\text { Vel. } \\
(\mathbf{r p m})\end{array}$ & $\begin{array}{c}\text { Avance } \\
(\mathbf{m m} / \\
\mathbf{r e v})\end{array}$ & $\begin{array}{c}\mathbf{R a} \\
(\mu \mathbf{m})\end{array}$ & Refrigeración \\
\hline Tipo H & 1.498 & 0,15 & 0,94 & Externa \\
\hline Tipo N & 1.498 & 0,15 & 0,88 & Externa \\
\hline Tipo W & 1.498 & 0,15 & 1,86 & Externa \\
\hline $\begin{array}{c}\text { MegaJet } \\
\text { A6292 }\end{array}$ & 1.498 & 0,20 & 0,58 & Interna \\
\hline $\begin{array}{c}\text { Garant } \\
12235\end{array}$ & 5.853 & 0,17 & 0,40 & Interna \\
\hline
\end{tabular}




\section{Roscado}

En estos ensayos se utilizaron machos Titex Plus M-10 con remoción de viruta B 1233, B 1234, y B 1251 con tres hélices, y el macho $\mathrm{B} 1235$ con dos hélices, utilizando las perforaciones realizadas con la broca $\mathrm{W}$ de $8,5 \mathrm{~mm}$ de diámetro.

Con un macho M-10, marca Emuge, para roscado por conformación sin desprendimiento de viruta se hizo una serie de ensayos. Previamente el material fue perforado con una broca con refrigeración interior de 9,3 $\mathrm{mm}$ de diámetro.

Medición de ángulos de corte de los machos. En la tabla 4 se muestran los ángulos de ataque $(\gamma)$, obtenidos por catálogo, y los ángulos de hélice $(\delta)$, obtenidos por medición de los distintos machos utilizados.

Los ángulos de ataque de los diferentes machos oscilan entre $9^{\circ}$ y $20^{\circ}$, siendo el macho B 1235 el que presentó el mayor ángulo con $20^{\circ}$.

Tabla 4. Ángulos de ataque obtenidos en la medición para los distintos machos.

\begin{tabular}{|c|c|c|}
\hline MACHOS & $\begin{array}{c}\text { Ángulo de } \\
\text { Ataque }(\gamma)\end{array}$ & $\begin{array}{c}\text { Ángulo de } \\
\text { Hélice }(\delta)\end{array}$ \\
\hline B 1233 & $16^{\circ}$ & $40^{\circ}$ \\
\hline B 1234 & $14^{\circ}$ & $40^{\circ}$ \\
\hline B 1235 & $20^{\circ}$ & $45^{\circ}$ \\
\hline B 1251 & $9^{\circ}$ & $35^{\circ}$ \\
\hline
\end{tabular}

En la figura 8 se muestran los diferentes valores para los machos con remoción de viruta.

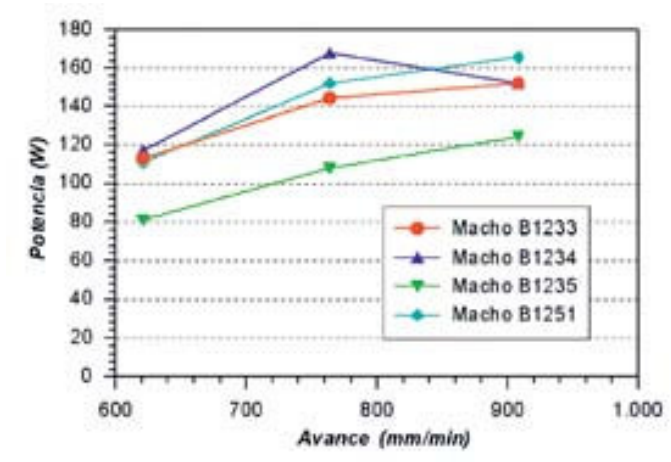

Figura 8. Potencia v/s Avance para machos con remoción de viruta.

Se observan que los consumos de potencia son similares, con excepción del macho B1235, que muestra los menores valores.
La figura 9 muestra las curvas de potencia para el macho por laminación marca Emuge.

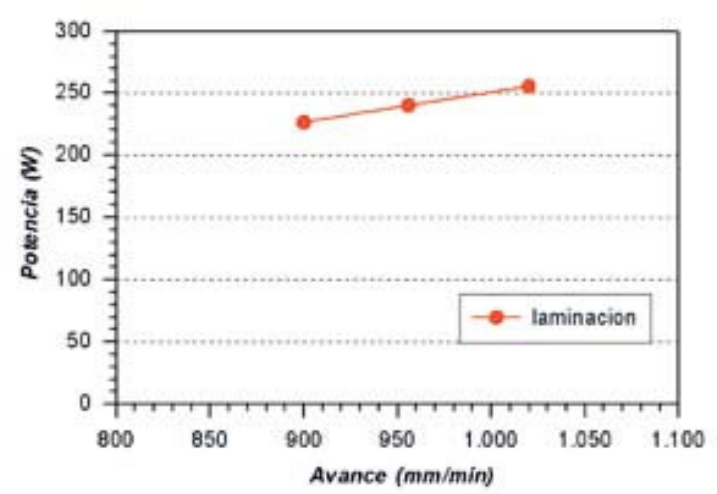

Figura 9. Potencia v/s Avance para el roscado por laminación.

Este macho trabaja con mayores velocidades de rotación que los machos convencionales, y el consumo de potencia es mayor.

\section{Ensayo de Tracción de las roscas M-10}

Las roscas realizadas fueron evaluadas con diferentes machos y fueron ensayadas en tracción. Para este ensayo se adaptó en la máquina universal de ensayos un dispositivo con ese objetivo. La tabla 5 entrega la fuerza máxima para cada rosca, después de colocar un perno con 6 vueltas, pasos o roscas, similar a una unión perno-tuerca.

Tabla 5. Resultados del ensayo de Tracción en roscas M-10 con seis pasos.

\begin{tabular}{|c|c|}
\hline MACHOS & $\begin{array}{c}\text { Fuerza Máxima } \\
{[\mathbf{N}]}\end{array}$ \\
\hline B 1233 & 32.365 \\
\hline B 1234 & 39.445 \\
\hline B 1235 & 41.773 \\
\hline B 1251 & 40.548 \\
\hline Laminado & 42.508 \\
\hline
\end{tabular}

La rosca menos resistente fue la confeccionada con el macho B 1233. Con los otros tres machos hay una variación de alrededor de un $8 \%$ entre ellos. La rosca más resistente fue la obtenida con el macho marca Emuge de roscado por laminación. La figura 10 muestra la probeta de ensayos y el perno de acero utilizado en los ensayos de tracción con la rosca de cobre arrancada. 


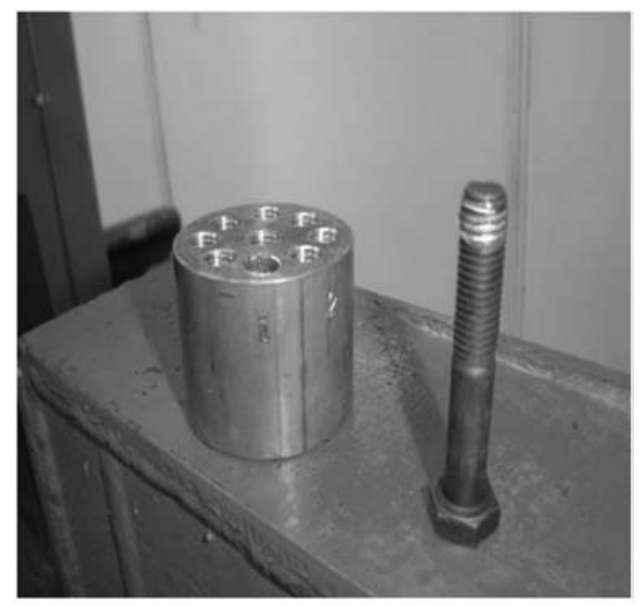

Figura 10. Probeta y perno de acero enroscado en 6 pasos.

\section{CONCLUSIONES}

De los ensayos experimentales con los tres tipos de brocas, se verifica que la broca tipo $\mathrm{W}$ es la que muestra el menor consumo de potencia, pero no se observa una variación significativa cuando opera con o sin refrigeración. La causa puede estar en el tamaño del agujero que es sólo de $8,5 \mathrm{~mm}$ de diámetro.

Este tipo de broca es la que presenta un ángulo de ataque muy próximo al ángulo de ataque efectivo obtenido experimentalmente a través de la teoría de Bill Mundy, lo que demuestra la validez de esta teoría en el perforado de cobre.

La calidad superficial de los agujeros depende directamente del sistema de refrigeración. Con las brocas con refrigeración interna se obtiene una rugosidad tres a cuatro veces menor que con refrigeración externa.

La falta de lubricación para la broca tipo $\mathrm{H}$ es crítica, ya que favorece el atascamiento e inclusive su quiebre durante la operación. Además el fabricante no recomienda esta broca para el taladrado de cobre.
En la operación de roscado el macho B 1235 de dos hélices mostró el menor consumo de potencia, con su ángulo de ataque de $20^{\circ}$ y ángulo de hélice de $45^{\circ}$. Nuevamente el ángulo de hélice es prácticamente el mismo que se obtiene con la teoría de Bill Mundy.

Todas las roscas obtenidas por corte o arranque de virutas con los machos Modelos B 1234, B 1235 y B 1251 resistieron fuerzas cercanas a 40.000 Newton.

La rosca confeccionada con el macho por laminación alcanzó una resistencia cercana de 42.000 Newton. Este aumento de resistencia se debe a la deformación del material durante el proceso de laminado por conformación. Este proceso entrega una rosca más resistente.

\section{AGRADECIMIENTOS}

Empresa Titex Plus, Frankfurt, Alemania.

Profesor Dr. Franz Tikal, Instituto de Producción y Logística de la Universidad de Kassel.

\section{REFERENCIAS}

[1] B. Mundy. "How does a metal want to be cut?". American Machinist \& Automated Manufacturing. July 1987.

[2] J.M. Godoy R., J. Vergara D., J. Villanueva A., M. Quispe Y., P. Oviedo O., F. Tikal and S. Damm. "Cutting Forces Measurements with a Piezoelectric Dynamometer During Termal Flow Drilling". Revista de la Facultad de Ingeniería - Universidad de Tarapacá. Vol. 11 Nº 1, pp. 65-69. Arica, Chile. 2003.

[3] E. Pauscksch. "Zerspantechnik". Ed. Vieweg. 1996. Germany.

[4] Titex Plus Tex-Titex Electronic Catalog. CD version 2002. URLs: www.titex.com 